\title{
ON THE PADÉ APPROXIMANTS ASSOCIATED WITH A POSITIVE DEFINITE POWER SERIES*
}

BY

H. S. WALL

1. Introduction. To every power series:

$$
\mathfrak{B}(z)=c_{0}-c_{1} z+c_{2} z^{2}-\cdots \quad\left(c_{0} \neq 0\right),
$$

and to every pair of numbers $m, n$ of the sequence $0,1,2,3, \cdots$, there corresponds uniquely a rational function:

$$
[m, n]=N_{m, n}(z) / D_{m, n}(z),
$$

in which the degrees of numerator and denominator do not exceed $n$ and $m$, respectively, and such that the formal power series

$$
\mathfrak{B}(z) D_{m, n}(z)-N_{m, n}(z)
$$

shall begin with the $(m+n+1)$ th or a higher power of $z$. The function (2) is called a Padé approximant $\dagger$ of $\mathfrak{B}(z)$.

Following Padé we shall form with the approximants the accompanying table of double entry:

\begin{tabular}{|c|c|c|c|}
\hline$[0,0]=c_{0}$ & {$[0,1]=c_{0}-c_{1} z$} & {$[0,2]=c_{0}-c_{1} z+c_{2} z^{2}$} & $\cdots$ \\
\hline$[1,0]$ & {$[1,1]$} & {$[1,2]$} & $\cdots$ \\
\hline$[2,0]$ & {$[2,1]$} & {$[2,2]$} & $\cdots$ \\
\hline & & \\
\hline
\end{tabular}

Let $S_{i}\left(S_{-i}\right), i>0$, designate the $i$ th diagonal file of approximants to the right of (below) and parallel to the principal diagonal, $S_{0}$, in (3). Then $S_{i}$ is the infinite sequence

$$
S_{i}=[0, i],[1, i+1],[2, i+2], \cdots ;
$$

and

$$
S_{-i}=[i, 0],[i+1,1],[i+2,2], \cdots .
$$

Both (4) and (5) give $S_{0}$ for $i=0$.

* Presented to the Society, March 29 and December 30, 1929; received by the editors December 30, 1929.

† For details see Perron, Die Lehre von den Kettenbrüchen, Leipzig and Berlin, 1913, Chapter X. 
Let

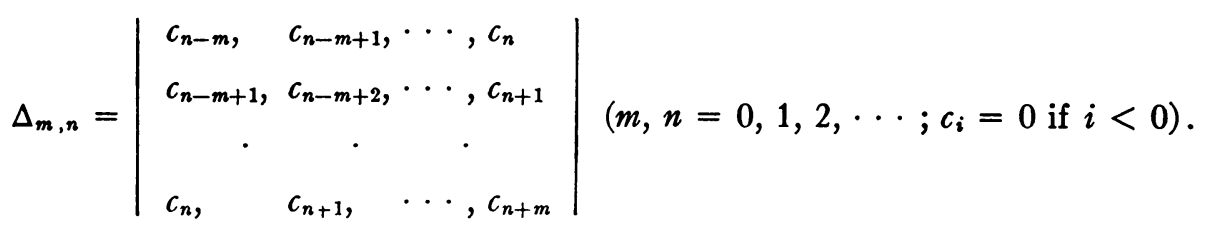

Then if

$$
\Delta_{n, n}>0 \quad(n=0,1,2, \cdots),
$$

$\mathfrak{B}(z)$ is called positive definite. The object of the following article is to study the convergence of the diagonal files, $S_{i}$, in the Padé table associated with a positive definite series, and to investigate the relationship among, and the character of, the limits (when they exist) of these files.* A summary of the principal results is contained in $\S 7$.

2. The polynomials $A_{n}^{k}, B_{n}^{k}$. With each of the series $\dagger$

$$
\mathfrak{P}^{k}(z)=c_{k}-c_{k+1} z+c_{k+2} z^{2}-\cdots
$$

we shall associate four polynomials, namely

$$
\begin{aligned}
& A_{2 n}^{k}=\alpha_{0}^{n, k}+\alpha_{1}^{n, k} z+\cdots+\alpha_{n-1}^{n, k} z^{n-1}, B_{2 n}^{k}=\beta_{0}^{n, k}+\beta_{1}^{n, k} z+\cdots+\beta_{n}^{n, k} z^{n}, \\
& A_{2 n+1}^{k}=\gamma_{0}^{n, k}+\gamma_{1}^{n, k} z+\cdots+\gamma_{n}^{n, k} z^{n}, \quad B_{2 n+1}^{k}=\delta_{0}^{n, k}+\delta_{1}^{n, k} z+\cdots+\delta_{n}^{n, k} z^{n},
\end{aligned}
$$

obtained by requiring that the formal power series

$$
\mathfrak{P}^{k} B_{m}^{k}-A_{\text {in }}^{k} \quad(m=2 n \text { or } 2 n+1)
$$

shall begin with the $m$ th power of $z$. It will be seen that this requirement yields the following systems of equations:

$$
\begin{array}{lr}
\sum_{i=0}^{p}(-1)^{i} c_{k+i} \beta_{p-i}^{n, k}=\alpha_{p}^{n, k} & (p=0,1, \cdots, n-1), \\
\sum_{i=0}^{p}(-1)^{i} c_{k+i} \delta_{p-i}^{n, k}=\gamma_{p}^{n, k} & (p=0,1, \cdots, n) ; \\
\sum_{i=0}^{n}(-1)^{i} c_{k+p+i} \beta_{n-i}^{n, k}=0 & (p=0,1, \cdots, n-1), \\
\sum_{i=0}^{n}(-1)^{i} c_{k+p+i} \delta_{n-i}^{n, k}=0 & (p=1,2, \cdots, n) .
\end{array}
$$

* Cf. Wall, these Transactions, vol. 31 (1929), pp. 91-110, in which the same question is studied under the further restriction that (1) shall be a series of Stieltjes.

$\dagger$ In $\S \S 2,3,4$ the series $\mathfrak{B}(z)$ is not restricted to be positive definite, but is an arbitrary series with constant term different from zero. 
Since the homogeneous systems (9) both possess fewer equations (by one) than unknowns they may be satisfied in every case by sets of values of the $\beta_{i}^{n, k}, \delta_{i}^{n, k}$, respectively, each set having at least one element not zero. The $\alpha_{i}^{n, k}, \gamma_{i}^{n, k}$ are then determined by the $\beta_{i}^{n, k}, \delta_{i}^{n, k}$, respectively, in accordance with (8).

Though the solution of (8), (9) is not unique, the functions $A_{m}^{k} / B_{m}{ }^{k}$ are unique provided the $\beta_{i}^{n, k}$ or the $\delta_{i}^{n, k}$, as the case may be, are not all zero. In fact, if the power series

$$
\begin{gathered}
\mathfrak{P}^{k} B_{m}^{k}-A_{m}^{k}, \\
\mathfrak{P}^{k} B_{m, 1}^{k}-A_{m, 1}^{k}
\end{gathered}
$$

both begin with the $m$ th or a higher power of $z$, the polynomial

$$
A_{m, 1}^{k} B_{m}^{k}-A_{m}^{k} B_{m, 1}^{k}
$$

which is identical with

$$
\left(\mathfrak{P}^{k} B_{m}^{k}-A_{m}^{k}\right) B_{m, 1}^{k}-\left(\mathfrak{P}^{k} B_{m, 1}^{k}-A_{m, 1}^{k}\right) B_{m}^{k},
$$

contains no power of $z$ lower than the $m$ th. But since it is of degree $m-1$ at most it must vanish identically, and therefore

$$
A_{m, 1}^{k} / B_{m, 1}^{k} \equiv A_{m}^{k} / B_{m}^{k} \text {. }
$$

We shall suppose in general that the polynomials (7) are determined by an arbitrary non-trivial solution of (9). Only when the determinant $\Delta_{n-1, n+k}$ is different from zero do we take a particular determination of the $A_{2 n}, B_{2 n}$, which is in fact unique apart from a common constant factor, namely

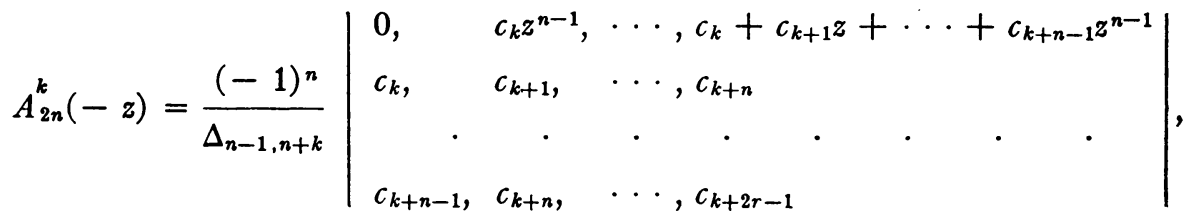

$$
\begin{aligned}
& B_{2 n}^{k}(-z)=\frac{(-1)^{n}}{\Delta_{n-1, n+k}}\left|\begin{array}{cc}
z^{n}, & z^{n-1}, \cdots, 1 \\
c_{k}, & c_{k+1}, \cdots, c_{k+n} \\
\cdot & \cdot \\
c_{k+n-1}, & c_{k+n}, \cdots, c_{k+2 n-1}
\end{array}\right| .
\end{aligned}
$$

If $\Delta_{n, n+k} \neq 0$, we shall take 


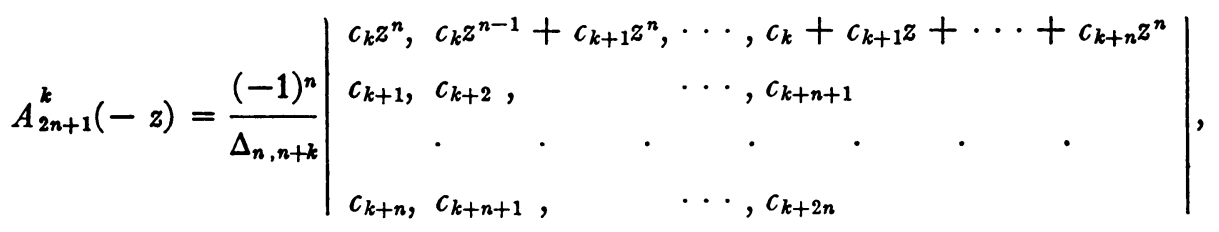

$$
B_{2 n+1}^{k}(-z)=\frac{(-1)^{n}}{\Delta_{n, n+k}}\left|\begin{array}{ccc}
z^{n}, & z^{n-1}, & \cdots, 1 \\
c_{k+1}, & c_{k+2}, & \cdots, \\
\cdot & \cdot & c_{k+n+1} \\
c_{k+n}, & c_{k+n+1}, \cdots, & \cdots
\end{array}\right|
$$

These are likewise unique except for a common constant factor.

3. Expressions for the $A_{n}^{k}, B_{n}^{k}$ in terms of the $A_{n}^{k-1}, B_{n}^{k-1}$. Let us assume that

$$
\Delta_{n-1, n+k-1}, \Delta_{n, n+k-1} \neq 0 .
$$

Then we shall determine constants $K_{1}, K_{2}$ such that

$$
\begin{gathered}
B_{2 n-1}^{k}=K_{1} B_{2 n}^{k-1}-K_{2} B_{2 n+1}^{k-1}, \\
z \cdot A_{2 n-1}^{k}=c_{k-1}\left(K_{1} B_{2 n}^{k-1}-K_{2} B_{2 n+1}^{k-1}\right)-\left(K_{1} A_{2 n}^{k-1}-K_{2} A_{2 n+1}^{k-1}\right) .
\end{gathered}
$$

The polynomials (7) involved in (13), (14) are given by (10), (11) by virtue of (12). If then we equate the coefficients of the highest and of the lowest powers of $z$ in (13) we shall find that

$$
K_{2}=1, K_{1}=\Delta_{n-1, n+k} / \Delta_{n, n+k-1} .
$$

One may then verify that the right member of (14) is divisible by $z$, and that the power series for $\mathfrak{B}^{k} B_{2 n-1}^{k}-A_{2 n-1}^{k}$ (using the values of $A_{2 n-1}^{k}, B_{2 n-1}^{k}$ from (13), (14)) begins with the $(2 n-1)$ th or a higher power of $z$.

In like manner, if

$$
\Delta_{n-1, n+k}, \Delta_{n, n+k-1} \neq 0
$$

one will find that

$$
B_{2 n}^{k}=K B_{2 n+1}^{k-1}, \quad z A_{2 n}^{k}=c_{k-1} K B_{2 n+1}^{k-1}-K A_{2 n+1}^{k-1},
$$

where $K=1 / K_{1}$.

We have proved the following theorem.

TheOREM 1. If (12) holds, then

$$
B_{2 n-1}^{k}=h_{n}^{k-1} B_{2 n}^{k-1}-B_{2 n+1}^{k-1}
$$




$$
z A_{2 n-1}^{k}=c_{k-1}\left[h_{n}^{k-1} B_{2 n}^{k-1}-B_{2 n+1}^{k-1}\right]-\left[h_{n}^{k-1} A_{2 n}^{k-1}-A_{2 n+1}^{k-1}\right]
$$

where

$$
h_{n}^{k-1}=\Delta_{n-1, n+k} / \Delta_{n, n+k-1} .
$$

If (15) holds, then

$$
\begin{aligned}
h_{n}^{k-1} B_{2 n}^{k} & =B_{2 n+1}^{k-1} . \\
z h_{n}^{k-1} A_{2 n}^{k} & =c_{k-1} B_{2 n+1}^{k-1}-A_{2 n+1}^{k-1} .
\end{aligned}
$$

4. The Padé approximants. Let $\mathfrak{B}_{k}$ denote the sum of the first $k$ terms of $\mathfrak{P}$, and consider the functions

$$
\begin{aligned}
F_{n}^{k} & =\left(\mathfrak{P}_{k} B_{2 n-1}^{k}+(-z)^{k} A_{2 n-1}^{k}\right) / B_{2 n-1}^{k}, \\
F_{n+1}^{k-1} & =\left(\mathfrak{B}_{k} B_{2 n}^{k}+(-z)^{k} A_{2 n}^{k}\right) / B_{2 n}^{k} \quad(n, k=1,2,3, \cdots) .
\end{aligned}
$$

The degrees of numerator and denominator of (21) do not exceed $n+k-1$, $n-1$, respectively. Then since the formal power series

$$
\mathfrak{B} B_{2 n-1}^{k}-\left(\mathfrak{B}_{k} B_{2 n-1}^{k}+(-z)^{k} A_{2 n-1}^{k}\right)=(-z)^{k}\left(\mathfrak{P}^{k} B_{2 n-1}^{k}-A_{2 n-1}^{k}\right)
$$

begins with the $(k+2 n-1)$ th or a higher power of $z$, it follows (see Introduction) that $F_{n}^{k}$ is the Padé approximant $[n-1, n+k-1]$ of $\mathfrak{B}$, i.e.,

$$
F_{n}^{k}=[n-1, n+k-1] \quad(n, k=1,2, \cdots) .
$$

Similarly, (22) is the Padé approximant $[n, n+k-1]$ of $\mathfrak{P}$.

Set $A_{m}{ }^{0} \equiv A_{m}, B_{m}{ }^{0} \equiv B_{m}$. Then clearly

$$
F_{n}=A_{2 n-1} / B_{2 n-1}, F_{n}^{-1}=A_{2 n} / B_{2 n} \quad(n=1,2, \cdots)
$$

are the approximants $[n-1, n-1],[n, n-1]$, respectively.

Let $C_{n}^{k}, D_{n}^{k}$ denote the polynomials $A_{n}^{k}, B_{n}^{k}$, respectively, connected with the reciprocal of $\mathfrak{B}$, namely

$$
r^{\xi}(z)=d_{0}-d_{1} z+d_{2} z^{2}-\cdots .
$$

If $\xi_{k}$ is the sum of the first $k$ terms, then

$$
\begin{aligned}
F_{n}^{-k} & =D_{2 n-1}^{k} /\left(\xi_{k} D_{2 n-1}^{k}+(-z)^{k} C_{2 n-1}^{k}\right), \\
F_{n+1}^{-k+1} & =D_{2 n}^{k} /\left(\xi_{k} D_{2 n}^{k}+(-z)^{k} C_{2 n}^{k}\right) \quad(n, k=1,2, \cdots)
\end{aligned}
$$


are the approximants $[n+k-1, n-1],[n+k-1, n]$, respectively, of $\mathfrak{B}$. Taking (25), for example, we see that the degrees of numerator and denominator do not exceed $n-1, n+k-1$, respectively, while the formal power series

$$
\mathfrak{P}\left(\xi_{k} D_{2 n-1}^{k}+(-z)^{k} C_{2 n-1}^{k}\right)-D_{2 n-1}^{k}=-(-z)^{k} \mathfrak{P}\left(\xi^{k} D_{2 n-1}^{k}-C_{2 n-1}^{k}\right)
$$

begins with at least the $(2 n+k-1)$ th power of $z$.

Along with (25), (26) we have

$$
F_{n}=D_{2 n-1} / C_{2 n-1}, F_{n}^{1}=D_{2 n} / C_{2 n} .
$$

5. The convergence of the diagonal files $S_{k}, k \geqq-1$, for a positive definite series. If $\mathfrak{B}(z)$ is positive definite, then the series $\mathfrak{P}^{2 k}(z), k=1,2,3, \cdots$, are all positive definite inasmuch* as the determinants $\Delta_{n, n+2 k}, n, k=0,1$, $2, \cdots$, are all positive if they are positive when $k=0$.

It is seen from the relation

$$
\Delta_{n, n+2 k+1} \Delta_{n-2, n+2 k+1}=\Delta_{n-1, n+2 k} \Delta_{n-1, n+2 k+2}-\Delta_{n-1, n+2 k+1}^{2}
$$

that for each $k=0,1,2, \cdots$, the determinants $\Delta_{n, n+2 k+1}, n=0,1,2, \cdots$, cannot vanish for two consecutive values of $n$. Let $\left(n^{\prime}\right)$ denote the set of all distinct indices $n$ for which $\Delta_{n-1, n+2 k} \neq 0$ ( $k$ being fixed). Then $\left(n^{\prime}\right)$ is infinite.

If $n_{1}$ is not in $\left(n^{\prime}\right)$ then the four approximants

$$
\begin{array}{ll}
{\left[n_{1}-1, n_{1}+2 k-1\right],} & {\left[n_{1}-1, n_{1}+2 k\right],} \\
{\left[n_{1}, n_{1}+2 k-1\right],} & {\left[n_{1}, n_{1}+2 k\right],}
\end{array}
$$

are identical. $\dagger$ No other approximants of the table are equal to these.

From these considerations we immediately deduce the following result (vide supra, (4), (23)).

Theorem 2. If $\mathfrak{B}(z)$ is positive definite and the limits

$$
\lim _{n=\infty} F_{n}^{2 k-1}=S_{2 k-1}, \lim _{n=\infty} F_{n}^{2 k}=S_{2 k}, \lim _{n=\infty} F_{n}^{2 k+1}=S_{2 k+1}
$$

exist and two (or all) of them are distinct for $z=z_{0}$, then there exists a number $N$ such that

$$
\Delta_{n-1, n+2 k} \neq 0 \text { if } n \geqq N .
$$

* Sylvester, Philosophical Magazine, (4), vol. 4 (1852), pp. 140-141.

$\dagger$ Perron, loc. cit., p. 426. Note that my $\Delta_{m, n}$ differs from that used by Perron by the factor \pm 1 . Van Vleck showed that all irregularities of the table must be of the first order. Cf. these Transactions, vol. 4 (1903), pp. 297-332; p. 330. 
In order to connect with Hamburger's work* on positive definite power series, we now set

$$
\begin{gathered}
P_{2 n}^{k}\left(z^{\prime}\right)=z^{\prime n-1} A_{2 n}^{k}(z), \quad Q_{2 n}^{k}\left(z^{\prime}\right)=z^{\prime n} B_{2 n}^{k}(z), \\
P_{2 n+1}^{k}\left(z^{\prime}\right)=z^{\prime n} A_{2 n+1}^{k}(z), Q_{2 n+1}^{k}\left(z^{\prime}\right)=z^{n+1} B_{2 n+1}^{k}(z),
\end{gathered}
$$

where $z^{\prime}=1 / z$. When (10), (11) hold, and $k$ is even, one will readily identify these polynomials with Hamburger's polynomials $P_{n}\left(z^{\prime}\right), Q_{n}\left(z^{\prime}\right)$ connected with the positive definite power series $\mathfrak{P}^{k}\left(z^{\prime}\right) / z^{\prime}\left(k\right.$ even). When $\Delta_{n-1, n+2 k}=0$, $P_{2 n}^{2 k}\left(z^{\prime}\right) / Q_{2 n}^{2 k}\left(z^{\prime}\right)$ is to be identified with Hamburger's $U_{n}\left(z^{\prime}\right) / V_{n}\left(z^{\prime}\right)$ (for the series $\left.\mathfrak{B}^{2 k}\left(z^{\prime}\right) / z^{\prime}\right)$.

Now by (21), (22), (31), (32),

$$
\begin{aligned}
F_{n}^{2 k} & =\mathfrak{P}_{2 k}(z)+z^{2 k-1} P_{2 n-1}^{2 k}\left(z^{\prime}\right) / Q_{2 n-1}^{2 k}\left(z^{\prime}\right), \\
F_{n+1}^{2 k-1} & =\mathfrak{P}_{2 k}(z)+z^{2 k-1} P_{2 n}^{2 k}\left(z^{\prime}\right) / Q_{2 n}^{2 k}\left(z^{\prime}\right),
\end{aligned}
$$

$k=0,1,2, \cdots\left(\mathfrak{B}_{0}=0, F_{n+1}^{-1}\right.$ to be replaced by $\left.F_{n}^{-1}\right)$. Then by (4), (23), (24), (33), and Theorem XIX of Hamburger's memoir, $\dagger$ we have immediately

THEOREM 3. If $\mathfrak{B}(z)$ is positive definite, then over every closed finite region of the z-plane which contains no part of the real axis,

where

$$
S_{2 k}=\lim _{n=\infty} F_{n}^{2 k}=\mathfrak{P}_{2 k}+z^{2 k-1} I^{2 k}\left(z^{\prime}\right) \quad(k=0,1,2, \cdots),
$$

$$
I^{2 k}\left(z^{\prime}\right)=\int_{-\infty}^{+\infty} \frac{d \psi^{2 k}(u)}{z^{\prime}+u},
$$

a Stieltjes integral in which $\psi^{2 k}(u)$ is a monotone non-decreasing real function $\phi(u)$ satisfying the equations

$$
c_{2 k+i}=\int_{-\infty}^{+\infty} u^{i} d \phi(u) \quad(i=0,1,2, \cdots) .
$$

The function $\ddagger \psi^{2 k}(u)$ is determined by the condition that it shall have a greater saltus at $u=0$ than every $\S$ other function $\phi(u)$ satisfying (35).

* Hans Hamburger, Mathematische Annalen, vol. 81 (1920), pp. 235-319, and vol. 82 (1921), pp. 120-187. This excellent memoir furnishes the basis for the present paper.

$\dagger$ Loc. cit., vol. 81, p. 310.

$\ddagger$ The "Maximalbelegungsfunktion" of Hamburger: cf. loc. cit., vol. 81, pp. 298-299, Theorem XVI and Definition XII.

$\S$ Two functions $\phi$ and $\phi_{1}$ will be considered identical if they agree, with the possible exception of an additive constant, at all points of continuity. 
We shall call the functions $\phi(u)$ satisfying (35) moment functions of $\mathfrak{P}^{2 k}$. In particular the moment function $\psi^{2 k}(u)$ defined in Theorem 3 is the maximal moment function of $\mathfrak{P}^{2 k}$.

There are two cases to be considered. Either the maximal moment function is the only moment function of $\mathfrak{P}^{2 k}$, or else $\mathfrak{P}^{2 k}$ has an infinite number of different moment functions. These will be called the determinate and the indeterminate cases, respectively, and it will be convenient to term $\mathfrak{P}^{2 k}$ determinate and indeterminate.

In the indeterminate case, ${ }^{*}$ the saltus of $\psi^{2 k}(u)$, the maximal moment function, is positive at $u=0$, i.e.,

$$
\lim _{\epsilon \rightarrow 0}\left[\psi^{2 k}(\epsilon)-\psi^{2 k}(-\epsilon)\right]=\delta>0, \epsilon>0,
$$

and in fact,

$$
\frac{1}{\delta} \equiv h^{2 k}=\lim _{n=\infty} h_{n}^{2 k}
$$

where $h_{n}^{2 k}$ is given by (18).

For the determinate case, the work of Hamburger $\dagger$ and (34), (33) give at once the following theorem:

THEOREM 4. If $\mathfrak{B}(z)$ is positive definite and $\mathfrak{P}^{2 k}$ determinate, then over every finite closed region containing no part of the real axis, $S_{2 k-1}$ converges uniformly and

Let us set

$$
S_{2 k-1} \equiv S_{2 k}
$$

$$
\stackrel{2 k}{g_{n^{\prime}}}=P_{2 n^{\prime}}^{2 k}(0) / Q_{2 n^{\prime}}^{2 k}(0), n^{\prime} \text { in }\left(n^{\prime}\right) \quad(k=0,1,2, \cdots) .
$$

Then according to Hamburger, $\ddagger$

$$
\begin{aligned}
& P_{2 n^{\prime}}^{2 k}\left(z^{\prime}\right)=\Omega_{n^{\prime}}^{2 k}\left(z^{\prime}\right)+g_{n^{\prime}}^{2 k} P_{2 n^{\prime}-1}^{2 k}\left(z^{\prime}\right), \\
& Q_{2 n^{\prime}}^{2 k}\left(z^{\prime}\right)=\Theta_{n^{\prime}}^{2 k}\left(z^{\prime}\right)+g_{n^{\prime}}^{2 k}\left({ }_{2 n^{\prime}-1}^{2 k}\left(z^{\prime}\right) \quad(k=0,1,2, \cdots),\right.
\end{aligned}
$$

where $\Omega_{n^{\prime}}^{2 k}, \Theta_{n^{\prime}}^{2 k}$ are polynomials defined for all $n$. In the indeterminate case, $\S$

$$
\begin{array}{ll}
\lim _{n=\infty} P_{2 n-1}^{2 k}\left(z^{\prime}\right)=p_{1}^{2 k}\left(z^{\prime}\right), & \lim _{n=\infty} Q_{2 n-1}^{2 k}\left(z^{\prime}\right)=q_{1}^{2 k}\left(z^{\prime}\right), \\
\lim _{n=\infty} \Omega_{n}^{2 k}\left(z^{\prime}\right)=\omega^{2 k}\left(z^{\prime}\right), & \lim _{n=\infty} \Theta_{n^{\prime}}^{2 k}\left(z^{\prime}\right)=\theta^{2 k}\left(z^{\prime}\right),
\end{array}
$$

* Hamburger, loc. cit., vol. 81 , p. 295 , Theorem XV, p. 263 , Definition III, and formulas (57), (58), pp. 262-263.

† Loc. cit., vol. 82, p. 144, Theorem XXII; vol. 81, p. 292, Theorem XIV, p. 289, Definition XI, and p. 290.

$\ddagger$ Loc. cit., vol. 82, p. 123, equations (10).

§ Loc. cit., vol. 82, pp. 139-141, Theorems XX and XXI. 
uniformly over every finite closed region. Here $p_{1}{ }^{2 k}, q_{1}{ }^{2 k}, \omega^{2 k}, \theta^{2 k}$ are entire transcendental functions of $z^{\prime}$ subject to the identity

$$
p_{1}^{2 k} \theta^{2 k}-q_{1}^{2 k} \omega^{2 k}=+1 \text {. }
$$

Let $Z$ be a constant or a function of $z$ and set

$$
m^{2 k}\left(z^{\prime}, Z\right)=\frac{\omega^{2 k}\left(z^{\prime}\right)+Z_{p_{1}^{2 k}}^{2 k}\left(z^{\prime}\right)}{\theta^{2 k}\left(z^{\prime}\right)+Z q_{1}^{2 k}\left(z^{\prime}\right)},
$$

$k=0,1,2,3, \cdots, m^{0}\left(z^{\prime}, Z\right) \equiv m\left(z^{\prime}, Z\right)$. We define this function for $|Z|=\infty$ as follows:

$$
m^{2 k}\left(z^{\prime}, \infty\right) \equiv p_{1}^{2 k}\left(z^{\prime}\right) / q_{1}^{2 k}\left(z^{\prime}\right) .
$$

Theorem 5. (a) If $\mathfrak{B}(z)$ is positive definite and $\mathfrak{P}^{2 k}(z)$ is indeterminate, then

$$
S_{2 k}=\mathfrak{B}_{2 k}+z^{2 k-1} m^{2 k}\left(z^{\prime}, \infty\right) \quad\left(k=0,1,2, \cdots ; \mathfrak{B}_{0}=0\right) .
$$

(b) Under the same conditions, the files $S_{2 k-1}, S_{2 k+1}$ converge if and only if

$$
\lim _{n \rightarrow=\infty}\left|g_{n^{\prime}}^{2 k}\right|=\infty
$$

or else

$$
\lim _{n \prime=\infty} g_{n^{\prime}} 2 k=g^{2 k}, g^{2 k} \text { finite, }
$$

and (30) hold. In the former case,

$$
S_{2 i-1} \equiv S_{2 k} \equiv S_{2 k+1}
$$

while in the latter,

(45) $S_{2 k-1}=\mathfrak{B}_{2 k}+z^{2 k-1} m^{2 k}\left(z^{\prime}, g^{2 k}\right), S_{2 k+1}=\mathfrak{P}_{2 k}+z^{2 k-1} m^{2 k}\left(z^{\prime}, g^{2 k}-z / h^{2 k}\right)$

(where $h^{2 k}$ is the positive constant given by (37)), and

$$
S_{2 k-1} \not \equiv S_{2 k} \not \equiv S_{2 k+1} \text {. }
$$

Part (a) is the direct consequence of (33), (40).

Now by (34), (39), (40),

$$
\lim _{n \prime=\infty} F_{n^{\prime}+1}^{2 k-1}=\mathfrak{P}_{2 k}+z^{2 k-1} m^{2 k}\left(z^{\prime}, \infty\right), \text { or } \mathfrak{P}_{2 k}+z^{2 k-1} m^{2 k}\left(z^{\prime}, g^{2 k}\right),
$$

according as (42), (43), respectively, holds. It is easily seen with the aid of (41) that the latter is different from $S_{2 k}$.

Again, by (21), (16), (17), the argument being $z$ throughout, 


$$
F_{n^{\prime}}^{2 k+1}=\mathfrak{B}_{2 k+1}-z^{2 k+1} A_{2 n^{\prime}-1}^{2 k+1} / B_{2 n^{\prime}-1}^{2 k+1}=\mathfrak{B}_{2 k}+z^{2 k}\left[\frac{h_{n^{\prime}}^{2 k} A_{2 n^{\prime}}^{2 k}-A_{2 n^{\prime}+1}^{2 k}}{h_{n^{\prime}}^{2 k} B_{2 n^{\prime}}^{2 k}-B_{2 n^{\prime}+1}^{2 k}}\right]
$$

which becomes, by (31), (32), (39),

$$
F_{n^{\prime}}^{2 k+1}=\Re_{2 k}+z^{2 k-1}\left[\frac{\Omega_{n^{\prime}}^{2 k}\left(z^{\prime}\right)+\left(g_{n^{\prime}}^{2 k} P_{2 n^{\prime}-1}^{2 k}\left(z^{\prime}\right)-z P_{2 n^{\prime}+1}^{2 k}\left(z^{\prime}\right) / h_{n^{\prime}}^{2 k}\right.}{\Theta_{n^{\prime}}^{2 k}\left(z^{\prime}\right)+\left(g_{n^{\prime}}^{2 k} Q_{2 n^{\prime}-1}^{2 k}\left(z^{\prime}\right)-z Q_{2 n^{\prime}+1}^{2 k}\left(z^{\prime}\right) / h_{n^{\prime}}^{2 k}\right.}\right] .
$$

Hence," by (40),

(48) $\lim _{n \prime=\infty} F_{n^{\prime}}^{2 k+1}=\mathfrak{P}_{2 k}+z^{2 k-1} m^{2 k}\left(z^{\prime}, \infty\right)$, or $\mathfrak{P}_{2 k}+z^{2 k-1} m^{2 k}\left(z^{\prime}, g^{2 k}-z / h^{2 k}\right)$,

according as (42), (43), respectively, holds. As before the latter is different from $S_{2 k}$.

Let $\left(n_{1}\right)$ be the set of all indices $n$ not in $\left(n^{\prime}\right)$. Then if $\left(n_{1}\right)$ is finite, we may clearly replace $n^{\prime}$ by $n$ in (47), (48) and hence in this case we have (44) or (45) according as (42), (43), respectively, holds.

If, on the other hand, $\left(n_{1}\right)$ is infinite, it follows from the identity of the approximants in the squares (28) that

$$
\lim _{n_{1}=\infty} F_{n_{1}+1}^{2 k-1}=\lim _{n_{1}=\infty} F_{n_{1}}^{2 k+1}=S_{2 k}
$$

and if the second limits in (47), (48) hold, $S_{2 k-1}, S_{2 k+1}$ surely diverge inasmuch as they both contain an infinite subsequence with the different limit $S_{2 k}$.

The completion of the proof of (46) is accomplished, again making use of (41), by showing that the difference of the functions (45) is not zero.

We prove next a theorem connecting the various files $S_{2 k}$.

ThEOREM 6. (a) If $\mathfrak{P}^{2 k}$ is determinate, then $\mathfrak{P}^{2 k-2 t}, t \leqq k$, is determinate and

$$
S_{2 k} \equiv S_{2 k-2 t} \text {. }
$$

(b) If $\mathfrak{P}^{2 k-2 t}, t \leqq k$, is indeterminate, then $\mathfrak{P}^{2 k}$ is indeterminate, and

$$
S_{2 k} \not \equiv S_{2 k-2 t} \text {. }
$$

To prove (a) we write, by Theorem 3 ,

$$
S_{2 k-2 t}=\mathfrak{B}_{2 k-2 t}+z^{2 k-2 t-1} I^{2 k-2 t}\left(z^{\prime}\right), S_{2 k}=\mathfrak{B}_{2 k}+z^{2 k-1} I^{2 k}\left(z^{\prime}\right) .
$$

But clearly

$$
I^{2 k-2 t}\left(z^{\prime}\right)=c_{2 k-2 t} z-c_{2 k-2 t+1} z^{2}+\cdots+z^{2 t} \int_{-\infty}^{+\infty} u^{2 t} d \psi^{2 k-2 t}(u) /\left(z^{\prime}+u\right) .
$$


Hence

$$
S_{2 k}-S_{2 k-2 t}=z^{2 k-1}\left[\int_{-\infty}^{+\infty} \frac{d \psi^{2 k}(u)}{z^{\prime}+u}-\int_{-\infty}^{+\infty} \frac{d \psi_{1}{ }^{2 k}(u)}{z^{\prime}+u}\right],
$$

where

$$
d \psi_{1}{ }^{2 k}(u)=u^{2 t} \cdot d \psi^{2 k-2 t}(u) .
$$

But $\psi_{1}^{2 k}(u)$ is a moment function of the determinate series $\mathfrak{B}^{2 k}$, and therefore

$$
\psi_{1}{ }^{2 k}(u)=\int_{-\infty}^{u} u^{2 t} d \psi^{2 k-2 t}(u) \text { and } \psi^{2 k}(u)=\int_{-\infty}^{u} d \psi^{2 k}(u)
$$

agree at all points of continuity and at the points $z=+\infty,-\infty$. It then follows from a known theorem* that the right member of (51) is identically zero. Thus we prove (49).

To prove that $\mathfrak{B}^{2 k-2 t}$ is determinate, suppose the contrary. Then $\mathfrak{B}^{2 k-2 t}$ has two different moment functions:

$$
\psi_{i}^{2 k-2 t}(u), i=0,1
$$

Therefore $\mathfrak{B}^{2 k}$ has the two different moment functions:

$$
\psi_{i}^{2 k}(u)=\int_{-\infty}^{u} u^{2 t} d \psi_{i}^{2 k-2 t}(u), i=0,1,
$$

which is contrary to hypothesis.

To prove (b), assume that $\mathfrak{B}^{2 k}$ is determinate. Then by (a), just proved, $\mathfrak{B}^{2 k-2 t}$ is determinate, contrary to hypothesis. Therefore $\mathfrak{P}^{2 k}$ must be indeterminate.

Again, by Theorem 3,

$$
\begin{aligned}
S_{2 k-2 t} & =\mathfrak{B}_{2 k}+z^{2 k-1} \int_{-\infty}^{+\infty} u^{2 t} d \psi^{2 k-2 t}(u) /\left(z^{\prime}+u\right), \\
S_{2 k} & =\mathfrak{B}_{2 k}+z^{2 k-1} I^{2 k}\left(z^{\prime}\right),
\end{aligned}
$$

and if these were identical it would follow that

$$
\psi^{2 k}(u)=\int_{-\infty}^{u} u^{2 t} d \psi^{2 k-2 t}(u)
$$

But this is impossible since if it held, $\psi^{2 k}(u)$ would have zero saltus at $u=0$. This contradicts (36).

* Perron, loc. cit., p. 367, Lemma 1. 
Theorem 7. (a) If $\mathfrak{B}(z)$ is indeterminate, then the files $S_{2 k-1}, k=0,1,2$, $3, \cdots$, all converge or else all diverge.

(b) A necessary and sufficient condition for convergence is that

$$
\lim _{n \prime=\infty}\left|g_{n^{\prime}}\right|=\infty
$$

or else

$$
\lim _{n \prime=\infty} g_{n^{\prime}}=g, g \text { finite, }
$$

and $\Delta_{n-1, n}=0$ for but a finite number of values of $n$.

(c) When the odd files are convergent, two successive files $S_{n}, S_{n+1}$ have limits which are meromorphic functions of $z^{\prime}=1 / z$ expressible in the form $u_{n}(z) / v_{n}(z), u_{n+1}(z) / v_{n+1}(z)$, where $u_{n}(z), v_{n}(z), \cdots$, are entire functions of $1 / z$ among which there is, in general, the relation

$$
u_{n}(z) v_{n+1}(z)-u_{n+1}(z) v_{n}(z)=(-z)^{n} .
$$

An exception arises whenever, for some $k$,

$$
\lim _{n \rightarrow=\infty}\left|g_{n^{\prime}}^{2 k}\right|=\infty
$$

whereupon

$$
S_{2 k-1} \equiv S_{2 k} \equiv S_{2 k+1} \text {. }
$$

The identity (53) cannot hold for two or more consecutive values of $k$. If $S_{2 k-1} \not \equiv S_{2 k}$ or $S_{2 k} \not \equiv S_{2 k+1}$, then $S_{2 k-1} \not \equiv S_{2 k} \not \equiv S_{2 k+1}$.

(d) $A$ sufficient condition for divergence of the odd files is that $\Delta_{n-1, n+2 k}=0$ for an infinite number of values of $n$ when $k=k_{1}$ and $k=k_{1}+1\left(k_{1} \geqq 0\right)$.

In fact, by Theorem 6 , all the series $\mathfrak{P}^{2 k}, k \geqq 0$, are indeterminate. Hence by Theorem 5 it is seen that if $S_{2 k-1}$ converges (diverges) then $S_{2 k+1}$ must converge (diverge), $k=0,1,2, \cdots$. Thus all the files $S_{2 k-1}$ converge or else they all diverge. The condition in (b) is obviously correct since it is a necessary and sufficient condition for the convergence of $S_{-1}$, by Theorem 5 .

The first part of (c) follows from Theorem 5 if we take

$$
v_{n}(z)=\theta^{2 k}\left(z^{\prime}\right)+g^{2 k} q_{1}^{2 k}\left(z^{\prime}\right), q_{1}^{2 k}\left(z^{\prime}\right), \text { or } \theta^{2 k}\left(z^{\prime}\right)+\left(g^{2 k}-z / h^{2 k}\right) q_{1}^{2 k}\left(z^{\prime}\right),
$$

according as $n=2 k-1,2 k$, or $2 k+1$, and then put

$$
u_{n}(z)=v_{n}(z)\left[\Re_{2 k}+z^{2 k-1} m^{2 k}\left(z^{\prime}, Z\right)\right] \text {, where } Z=g^{2 k}, \infty \text {, or } g^{2 k}-z / h^{2 k},
$$

according as $n=2 k-1,2 k$, or $2 k+1$. The relation (52) then follows from (41) after an easy calculation. 
That (53) cannot hold for two consecutive values of $k$ follows at once from (50). The last statement in (c) follows from Theorem 5.

Under the hypothesis of (d), it would follow from Theorem 2 that $S_{2 k_{1}} \equiv S_{2 k_{1}+2}$, again contradicting (50).

6. The convergence of the files $S_{-k}, k \geqq 1$, for a positive definite series. To investigate the convergence of the files $S_{-k}$, we turn to the reciprocal series $\&(z)$. The following theorem is fundamental.

THEOREM 8. If $\mathfrak{B}(z)$ is a determinate (indeterminate) positive definite power series, then* $-\xi^{2}(z)$ is a determinate (indeterminate) positive definite power series.

On the supposition that $\mathfrak{B}$ has a corresponding continued fraction of the form

$$
\frac{1}{a_{1}}+\frac{z}{a_{2}}+\frac{z}{a_{3}}+\cdots,
$$

a condition equivalent to (6) is

$$
a_{2 i+1}>0 \quad(i=0,1,2, \cdots) .
$$

Now - ${ }^{1}(z)$ will have a corresponding continued fraction $\dagger$

$$
\frac{1}{a_{2}}+\frac{z}{a_{3}}+\frac{z}{a_{4}}+\cdots,
$$

and consequently if

$$
g_{n}=a_{2}+a_{4}+\cdots+a_{2 n} \neq 0 \quad(n=1,2,3, \cdots),
$$

- $\mathfrak{F}^{2}(z)$ will have the corresponding continued fraction $\ddagger$

where

$$
\frac{1}{e_{1}}+\frac{z}{e_{2}}+\frac{z}{e_{3}}+\cdots,
$$

$$
e_{2 i+1}=a_{2 i+3}\left(g_{i+1}\right)^{2}, \quad e_{2 i}=a_{2 i+2} /\left(g_{i} g_{i+1}\right) .
$$

Hence by (55), (56), $e_{2 i+1}>0$, and therefore $-5^{2}$ is positive definite in this restricted case.

Under the same restrictions, if $\mathfrak{P}$ is indeterminate, then - $\mathbb{E}^{2}$ is indeterminate, and conversely. To verify this it is sufficient $\S$ to prove the convergence of the two following series:

* Here we write $\xi^{2}$ for $\xi^{(2)}$.

$\dagger$ Wall, loc. cit., p. 99. Theorem 1.

‡ Wall, loc. cit., pp. 102-103, formulas (49), (50).

\$ Hamburger, loc. cit., vol. 82, p. 148, Theorem XXIV. 

(A) $\sum e_{2 i+1}$,
(B) $\sum e_{2 i+1}\left(e_{2}+e_{4}+\cdots+e_{2 i}\right)^{2}$,

on the hypothesis that the like series connected with $\mathfrak{B}$, namely
(A) $\sum a_{2 i+1}$,
(B) $\sum a_{2 i+1}\left(a_{2}+a_{4}+\cdots+a_{2 i}\right)$,

converge; and conversely.

Clearly (58) (A) converges, being by (57) the same as (59) (B), which we now suppose convergent. Now, by (57),

$$
\begin{aligned}
\sum_{i=1}^{n} e_{2 i+1}\left(e_{2}+e_{4}+\cdots+e_{2 i}\right)^{2}=\frac{1}{\left(a_{2}\right)^{2}} \sum_{i=1}^{n} a_{2 i+3}\left(g_{i+1}\right)^{2} & \\
& -\frac{2}{a_{2}} \sum_{i=1}^{n} a_{2 i+3} g_{i+1}+\sum_{i=1}^{n} a_{2 i+8},
\end{aligned}
$$

from which the convergence of (58) (B) follows on the hypothesis that (59) converge, since $\sum a_{2 i+3} g_{i+1}$ converges then as Hamburger showed.*

Conversely, let (58) be convergent. Then as before (59) (B) converges. Now $\sum e_{2 i+1}\left(e_{2}+e_{4}+\cdots+e_{2 i}\right)$ is convergent, and, by (57),

$$
\sum_{i=1}^{n} e_{2 i+1}\left(e_{2}+e_{4}+\cdots+e_{2 i}\right)=\frac{1}{a_{2}} \sum_{i=1}^{n} a_{2 i+3}\left(g_{i+1}\right)^{2}-\sum_{i=1}^{n} a_{2 i+3} g_{i+1} .
$$

Multiplying the members of (61) by $2 / a_{2}$ and subtracting the resulting equation from (60), we get

$$
\begin{aligned}
\sum_{i=1}^{n} a_{2 i+3}= & \sum_{i=1}^{n} e_{2 i+1}\left(e_{2}+e_{4}+\cdots+e_{2 i}\right)^{2} \\
& -\frac{2}{a_{2}} \sum_{i=1}^{n} e_{2 i+1}\left(e_{2}+e_{4}+\cdots+e_{2 i}\right)^{2}+\frac{1}{\left(a_{2}\right)^{2}} \sum_{i=1}^{n} a_{2 i+3}\left(g_{i+1}\right)^{2} .
\end{aligned}
$$

Since the sums on the right of (62) converge for $n=\infty$, the like is true of the sum on the left, and hence (59) (A) converges.

Let now $\overline{\mathfrak{B}}(z)$ be obtained from $\mathfrak{B}(z)$ in the following manner. Suppose $t$ real, and write

$$
\frac{c_{0}}{\left(z^{\prime}+t\right)}-\frac{c_{1}}{\left(z^{\prime}+t\right)^{2}}+\frac{c_{2}}{\left(z^{\prime}+t\right)^{3}}-\cdots=\frac{\bar{c}_{0}}{z^{\prime}}-\frac{\bar{c}_{1}}{z^{\prime 2}}+\frac{\bar{c}_{2}}{z^{\prime 3}}-\cdots,
$$

where the series on the right is obtained by expanding in descending powers of $z^{\prime}$ each term of the series on the left and then collecting the like powers of $1 / z^{\prime}$. Then we set

$$
\overline{\mathfrak{B}}(z)=\bar{c}_{0}-\bar{c}_{1} z+\bar{c}_{2} z^{2}-\cdots .
$$

\footnotetext{
* Loc. cit., vol. 82 , p. 139 , formula (36).
} 
Lemma. If $\mathfrak{B}(z)$ is an indeterminate (determinate) positive definite power series, then if $t$ is real $\overline{\mathfrak{B}}(z)$ is an indeterminate (determinate) positive definite power series. There exists in every real interval an uncountable infinity of numbers $t$ such that $\overline{\mathfrak{B}}(z)$ will have a corresponding continued fraction

in which

$$
\frac{1}{\bar{a}_{1}}+\frac{z}{\bar{a}_{2}}+\frac{z}{\bar{a}_{3}}+\cdots
$$

$$
\bar{a}_{2}+\bar{a}_{4}+\cdots+\bar{a}_{2 i} \neq 0 \quad(i=1,2, \cdots) .
$$

The series $\mathbb{F}^{2}$ connected with $\overline{\mathfrak{B}}$ is $\overline{\mathfrak{F}}^{2}$, i.e., bears the same relationship to $\mathfrak{F}^{2}$ that $\overline{\mathfrak{P}}$ bears to $\mathfrak{B}$.

With the aid of this lemma the completion of the proof of our theorem may be accomplished. In fact, if $\mathfrak{B}$ fails to have a corresponding continued fraction, or if, while having a corresponding continued fraction, (56) fails, then we may turn to $\overline{\mathfrak{P}}$ in which $t$ has been chosen so that (63) exists and (64) holds. By what we have already proved, $-\overline{\mathbb{F}}^{2}$ will then be positive definite and determinate (indeterminate) if $\overline{\mathfrak{P}}$ and hence $\mathfrak{B}$ are of the same character. The same will then be true of $-\$^{2}$ since the latter is obtainable from $-\overline{\mathbb{F}}^{2}$ on subjecting it to the above described transformation, using $-t$ as the parameter.

Now that part of the lemma up to and including (63) was proved by Hamburger.* He showed that the function $P_{2 n}\left(z^{\prime}\right) / Q_{2 n}\left(z^{\prime}\right)$ connected with $\overline{\mathfrak{B}}(z)$ is equal $\dagger$ to $P_{2 n}\left(z^{\prime}+t\right) / Q_{2 n}\left(z^{\prime}+t\right)$. It follows that the sum (64) is equal to $P_{2 n}(t) / Q_{2 n}(t)$, and this will be different from 0 for all $n$ if we do not take $t$ to be one of the countable number of zeros of the polynomials $P_{2 n}(t)$, and this is clearly possible inasmuch as we have at our disposal an uncountable num. ber of choices for $t$. Now since

it follows that

$$
1 / \mathfrak{P}(z)=\mathfrak{E}(z),
$$

$$
1 /\left[\frac{c_{0}}{\left(z^{\prime}+t\right)}-\frac{c_{1}}{\left(z^{\prime}+t\right)^{2}}+\cdots\right]=d_{0} z^{\prime}-\left[d_{1}-d_{0} t\right]+\frac{\overline{d_{2}}}{z^{\prime}}-\frac{\overline{d_{3}}}{z^{\prime 2}}+\cdots .
$$

Thus

$$
\overline{\mathbb{E}}(z)=d_{0}-\left(d_{1}-d_{0} t\right) z+\bar{d}_{2} z^{2}-\bar{d}_{3} z^{3}+\cdots,
$$

and hence

$$
\bar{\S}^{2}(z)=\bar{d}_{2}-\bar{d}_{3} z+\bar{d}_{4} z^{2}-\cdots,
$$

* Loc. cit., vol. $81, \$ 4$, and p. 300 .

† Hamburger, loc. cit., vol. $81, \S 4$, p. 265 , formula (64). 
which was to be proved.

By (25), (26) we may now write

$$
\begin{aligned}
F_{n}^{-2 k} & =1 /\left[\xi_{2 k}+z^{2 k-1} P_{2 n-1}^{-2 k}\left(z^{\prime}\right) / Q_{2 n-1}^{-2 k}\left(z^{\prime}\right)\right], \\
F_{n+1}^{-2 k+1} & =1 /\left[\xi_{2 k}+z^{2 k-1} P_{2 n}^{-2 k}\left(z^{\prime}\right) / Q_{2 n}^{-2 k}\left(z^{\prime}\right)\right], \quad k \geqq 1,
\end{aligned}
$$

where $P_{n}{ }^{-2 k}, Q_{n}{ }^{-2 k}$ are related to $C_{n}^{2 k}, D_{n}^{2 k}$ by equations of the form (31), (32).

Theorems exactly analogous to the theorems of $\$ 4$ can now be stated for the files $S_{-k}, k \geqq 1$, using (65), (66) and Theorem 8 as our point of departure. It is important to observe that when $\mathfrak{B}(z)$ is indeterminate, the convergence or divergence of the file $S_{-1}$ implies the convergence or divergence, respectively, of all the other files $S_{k}$ where $k$ is odd.

7. Summary. From the preceding discussion one will readily see that there are just three cases, as follows.

Case I. When $\mathfrak{P}$ is indeterminate, all the even files of the table are convergent, and the limits are meromorphic functions of $z^{\prime}=1 / z$, such that, for every $i \geqq 0, k \geqq 1$,

$$
S_{2 i} \not \equiv S_{2 i+2 k}, S_{-2 i-2} \not \equiv S_{-2 i-2 k-2} \text {. }
$$

The odd files either all converge or else all diverge, and when convergent the limits of adjacent files of the table are, in general, unequal. In exceptional cases we may have

$$
S_{2 i-1} \equiv S_{2 i} \equiv S_{2 i+1} \quad(i=0, \pm 1, \pm 2, \cdots),
$$

but if this hold for $i=i^{\prime}, i^{\prime \prime}$, then $\left|i^{\prime}-i^{\prime \prime}\right|>1$.

Case II. The series $\mathfrak{P}^{2 k}$ and $-\mathbb{F}^{2 k^{\prime}}$ for $k=0,1,2, \cdots, p ; k^{\prime}=1,2$, $3, \cdots, p^{\prime}$, are determinate, while for $k>p, k^{\prime}>p^{\prime}$, respectively, these series are indeterminate. In this case the files $S_{i}, i=0,1, \cdots, 2 p ;-1,-2, \cdots$, $-2 p^{\prime}$, all converge to a common limit. For the remaining files in the upper and lower halves of.the table taken separately, the discussion is essentially the same as in Case I.*

Case III. The series $\mathfrak{B}$ and the associated series $\mathfrak{P}^{2 k},-\mathbb{C}^{2 k}$ are all determinate. Then all the files of the table have a common limit which is a function that is analytic over the entire plane except the whole or a part of the real axis.

In Case I the limits of the files $S_{i}, i \geqq-1$, are meromorphic functions of $1 / z$ with poles on the real axis only. As for $S_{-2}, S_{-3}, S_{-4}, \cdots$, the limits are

\footnotetext{
* See $\$ 9$ for further discussion of Case II.
} 
of the form $1 /\left[d_{0}-d_{1} z+z^{2} f(z)\right]$, where $f(z)$ is a meromorphic function of $1 / z$ with poles on the real axis only, and therefore these limits are meromorphic functions of $1 / z$ with zeros on the real axis only.

8. On extended positive definite series. In order to continue the discussion of the diagonal files we shall need to consider the question of "extending" positive definite series. We make the following definition: The positive definite power series $P(z)$ will be said to admit of an extension of order $n$ if there exist $2 n$ real numbers, $c_{-1}, c_{-2}, \cdots, c_{-2 n}$, such that the power series

$$
\mathfrak{P}^{(-2 i)}(z)=c_{-2 i}-c_{-2 i+1} z+\cdots \quad(i=1,2,3, \cdots, n)
$$

shall all be positive definite.

Two cases will be distinguished according as $\mathfrak{B ( z )}$ is determinate or indeterminate. In the former case we have the following theorem:*

THEOREM 9. Let $\mathfrak{B}(z)$ be a determinate positive definite power series with moment function $\psi(u)$ (cf. §5). Then a necessary and sufficient condition that $\mathfrak{B}(z)$ shall admit of an extension (67) of order $n$ is that the integral

$$
\int_{-\infty}^{+\infty} \frac{d \psi(u)}{u^{2 n}}
$$

shall be convergent. The coefficients $c_{-i}$ in an extension are as follows:

$$
c_{-i}=\int_{-\infty}^{+\infty} d \psi(u) / u^{i}, i<2 n ; \quad c_{-2 n} \geqq \int_{-\infty}^{+\infty} d \psi(u) / u^{2 n} .
$$

No other extensions of order $n$ are possible.

To prove that the convergence of (68) is sufficient for the existence of an extension of $\mathfrak{B}(z)$ of order $n$, we write

$$
\psi_{1}(u)=\int_{-\infty}^{u} \frac{d \psi(u)}{u^{2 n}},
$$

and note that this is a non-decreasing real function of $u$ such that all the moments

$$
c_{i}^{\prime}=\int_{-\infty}^{+\infty} u^{i} d \psi_{1}(u) \quad(i=0,1,2, \cdots)
$$

exist. Consequently, $\dagger$ the series

$$
c_{0}^{\prime}-c_{1}^{\prime} z+c_{2}^{\prime} z^{2}-\cdots
$$

* Cf. the corresponding theorem for Stieltjes series given by the writer in these Transactions, vol. 31, pp. 771-781.

† Hamburger, loc. cit., vol. 81, §5, pp. 266-270. 
is positive definite. But

$$
c_{2 n+i}^{\prime}=c_{i} \quad(i=0,1,2, \cdots),
$$

and therefore (70) is an extension of $\mathfrak{B}(z)$ of order $n$.

To prove the necessity of the condition, assume that an extension (67) of $\mathfrak{P}(z)$ exists. Clearly, since $\mathfrak{P}(z)$ is determinate, $\mathfrak{P}^{(-2 n)}(z)$ is determinate by Theorem 6. Let $\psi^{(-2 n)}(u)$ be the moment function of $\mathfrak{B}^{(-2 n)}(z)$. Then $\psi(u)$ and $\psi_{2}(u)=\int_{-\infty}^{u} u^{2 n} d \psi^{(-2 n)}(u)$ are both moment functions of the determinate series $\mathfrak{B}(z)$, and are therefore equal, with the possible exception of an additive constant, at all points of continuity. Thus if $-a<0, b>0, i \geqq 0,-a, b$ points of continuity of $\psi(u)$,

$$
\begin{aligned}
& \int_{-\infty}^{-a} \frac{d \psi(u)}{u^{2 n-i}}=\int_{-\infty}^{-a} u^{i} d \psi^{(-2 n)}(u), \\
& \int_{b}^{\infty} \frac{d \psi(u)}{u^{2 n-i}}=\int_{b}^{\infty} u^{i} d \psi^{(-2 n)}(u) .
\end{aligned}
$$

Now if $a, b$ approach 0 over points of continuity of $\psi(u)$, the right members of (71), (72), and therefore the left members, will approach definite limits, $L_{i}$, $L_{i}^{\prime}$, respectively. Let $-a^{\prime}$ be an arbitrary real number subject to the condition $0<a^{\prime}<\delta$, and let $-a_{1},-a_{2}$ be points of continuity of $\psi(u)$ such that $-\delta<-a_{1}<-a^{\prime}<-a_{2}<0$. Then if $\epsilon>0$, and $i$ is even,

$$
L_{i}-\frac{\epsilon}{2}<\int_{-\infty}^{-a_{1}} \frac{d \psi(u)}{u^{2 n-i}} \leqq \int_{-\infty}^{-a^{\prime}} \frac{d \psi(u)}{u^{2 n-i}} \leqq \int_{-\infty}^{-a_{2}} \frac{d \psi(u)}{u^{2 n-i}} \leqq L_{i},
$$

if $\delta$ is sufficiently small; if $i$ is odd, (73) is to be replaced by

$$
L_{i}+\frac{\epsilon}{2}>\int_{-\infty}^{-a_{1}} \frac{d \psi(u)}{u^{2 n-i}} \geqq \int_{-\infty}^{-a^{\prime}} \frac{d \psi(u)}{u^{2 n-i}} \geqq \int_{-\infty}^{-a_{2}} \frac{d \psi(u)}{u^{2 n-i}} \geqq L_{i} .
$$

Hence, whether $i$ is even or odd,

$$
\left|L_{i}-\int_{-\infty}^{-a^{\prime}} \frac{d \psi(u)}{u^{2 n-i}}\right|<\frac{\epsilon}{2} \quad\left(0<a^{\prime}<\delta\right) .
$$

Similarly,

$$
\left|L_{i}^{\prime}-\int_{b^{\prime}}^{+\infty} \frac{d \psi(u)}{u^{2 n-i}}\right|<\frac{\epsilon}{2} \quad\left(0<b^{\prime}<\delta^{\prime}\right) .
$$

It follows, if we combine (74), (75), that

$$
\int_{-\infty}^{+\infty} \frac{d \psi(u)}{u^{2 n-i}}, \quad i \geqq 0
$$


converges, and its limit is

$$
L_{i}+L_{i}^{\prime}=C_{-i}, \quad 2 n \geqq i \geqq 0 .
$$

When $i=2 n, L_{i}=\psi^{(-2 n)}(-0)$ while $L_{i}^{\prime}=c_{-2 n}-\psi^{(-2 n)}(+0)$, and hence in this case

$$
\int_{-\infty}^{+\infty} \frac{d \psi(u)}{u^{2 n}}=c_{-2 n}-\omega
$$

where $\omega \geqq 0$ is the saltus of $\psi^{(-2 n)}(u)$ at $u=0$.

When $\mathfrak{P}(z)$ is indeterminate, we proceed as follows. Set

$$
m\left(z^{\prime} ; t\right)=\frac{\omega\left(z^{\prime}\right)+t p_{1}\left(z^{\prime}\right)}{\theta\left(z^{\prime}\right)+t q_{1}\left(z^{\prime}\right)}, z^{\prime}=1 / z,
$$

where $t$ is a real parameter, and $\omega\left(z^{\prime}\right), \theta\left(z^{\prime}\right), \cdots$, are the functions introduced in (40). Hamburger* has shown that $m\left(z^{\prime} ; t\right)$ is a meromorphic function of $z^{\prime} \dagger$, which may be expressed as a Stieltjes integral:

$$
m\left(z^{\prime} ; t\right)=\int_{-\infty}^{+\infty} \frac{d \psi(u ; t)}{z^{\prime}+u}
$$

the latter having the asymptotic development $\mathfrak{P}\left(1 / z^{\prime}\right) / z^{\prime}$.

For any given finite value of $t, \psi(u ; t)$ is constant in the neighborhood of $u=0$, and hence all the moments

$$
c_{-i}=\int_{-\infty}^{+\infty} d \psi(u ; t) / u^{i} \quad(i=1,2, \cdots)
$$

exist. Thus if we set

$$
\psi^{(-2 n)}(u)=\int_{-\infty}^{u} d \psi(u ; t) / u^{2 n}
$$

where $n$ is an arbitrary positive integer, we see that $\psi^{(-2 n)}(u)$ is a non-decreasing function of $u$ such that all the moments

$$
c_{-2 n+i}=\int_{-\infty}^{+\infty} u^{i} d \psi^{(-2 n)}(u) \quad . \quad(i=0,1,2, \cdots)
$$

exist. Hence $\mathfrak{B}^{(-2 n)}(z)=c_{-2 n}-c_{-2 n+1} z+\cdots$ is a positive definite power series and is an extension of $\mathfrak{B}(z)$ of order $n$.

Hamburger $\ddagger$ showed that $\mathfrak{B}^{(-2)}(z)$ is determinate. It then follows from

* Loc. cit., vol. 82, §\$18-19.

$\dagger$ The $z^{\prime}$ of the preceding work.

‡ Loc. cit. vol. 82, p. 179. 
Theorem 6 that $\mathfrak{P}^{(-2 n)}(z)$ is determinate for $n=1,2, \cdots$. Also, $M+\mathfrak{P}^{(-2)}(z)$, $M>0$, is indeterminate, and it may in turn be extended, giving an extension $\mathfrak{P}_{1}(-4)(z)$ of $\mathfrak{B}(z)$ of order 2 which may be determinate or indeterminate, and is in any case different from $\mathfrak{B}^{(-4)}(z)$. This new series, $\mathfrak{P}_{1}{ }^{(-4)}(z)$, may then be extended, etc. We sum up these facts in the following theorem. The formulas (72) are given by Hamburger.

THEOREM 10. Let $\mathfrak{\beta}(z)$ be an indeterminate positive definite power series, and let $m(z ; t)$ be the meromorphic function of $z$ defined by (76), (77), which gives rise to a moment function $\psi(u ; t)$ of $\mathfrak{B}(z)$. Then $\mathfrak{B}(z)$ admits of a determinate extension of arbitrary order $n$ corresponding to every finite value of the real parameter $t$. The coefficients in the extension are given by (78).

The first two coefficients, $c_{-1}, c_{-2}$, are polynomials in $t$ as follows:*

$$
\begin{aligned}
& c_{-1}=t, \\
& c_{-2}=q_{1}^{\prime}(0) t^{2}+\left[\theta^{\prime}(0)-p_{1}^{\prime}(0)\right] t-\omega^{\prime}(0) .
\end{aligned}
$$

The series $\left(M+c_{-2}\right)-c_{-1} z+c_{0} z^{2}-\cdots, M>0$, is positive definite and indeterminate. This series may then be extended to any desired order, forming thereby, at pleasure, extensions of $\mathfrak{B}(z)$ all of which are indeterminate, or else such that all from and after a certain order are determinate.

Let us suppose that there exists a sequence of indices

such that (cf. (38))

$$
n_{1}<n_{2}<n_{3}<\cdots
$$

$$
\lim _{p \rightarrow \infty} g_{n_{p}}^{2 i}=g^{2 i},
$$

where $g^{2 i}$ is finite. Then by (34), (39), (40), (23), the sequence of approximants in $S_{2 i-1}$,

$$
\left[n_{p}, n_{p}+2 i-1\right] \quad(p=1,2, \cdots),
$$

will have the limit $c_{0}-c_{1} z+\cdots-c_{2 i-1} z^{2 i-1}+z^{2 i-1} m^{2 i}\left(z^{\prime}, g^{2 i}\right)$.

Let $\psi^{2 i}(u, t)$ be the moment function of $\mathfrak{P}^{2 i}$ occurring in the expression (76) for $m^{2 i}\left(z^{\prime}, g^{2 i}\right)$, and set

$$
\int_{-\infty}^{+\infty} \frac{d \psi^{2 i}\left(u, g^{2 i}\right)}{u^{k}}=c_{k}^{(i)} \quad(k=1,2,3, \cdots) .
$$

Then we shall show that the series

$$
\frac{c_{1}^{(i)}}{z}-\frac{c_{2}^{(i)}}{z^{2}}+\frac{c_{3}^{(i)}}{z^{3}}-\cdots
$$

* The primes denote differentiation with respect to $z$. 
converges outside a circle of radius $R$ where $R$ is the distance from the origin to the most remote pole of $m^{2 i}\left(z^{\prime}, g^{2 i}\right) / z$ (and is necessarily finite).

In fact, $\psi^{2 i}\left(u, g^{2 i}\right)$ is constant in the interval $\left(-R^{-1},+R^{-1}\right)$ and hence, if $R>\sigma>0$,

$$
\begin{aligned}
\frac{m^{2 i}\left(z^{\prime}, g^{2 i}\right)}{z} & =\int_{-\infty}^{+\infty} \frac{d \psi^{2 i}\left(u, g^{2 i}\right)}{1+z u}=\int_{-R \rightarrow \sigma}^{R+\sigma} \frac{-u d \psi^{2 i}\left(1 / u, g^{2 i}\right)}{z+u} \\
& =\int_{-R-\sigma}^{R+\sigma}-u\left\{\frac{1}{z}-\frac{u}{z^{2}}+\cdots\right\} d \psi^{2 i}\left(1 / u, g^{2 i}\right) .
\end{aligned}
$$

Since the series within the braces converges uniformly over the interval $-R-\sigma \leqq u \leqq R+\sigma$, if $|z|>R+\sigma$, it may be integrated term by term and hence (81) converges for $|z|>R$ and is equal to $m^{2 i}\left(z^{\prime}, g^{2 i}\right) / z$ there. We state the following theorem.

TheOREM 11. Let $\mathfrak{B}(\mathrm{z})$ be indeterminate, and suppose $S_{2 i-1}$ contains an infinite subsequence of approximants:

$$
\left[n_{p}, n_{p}+2 i-1\right] \quad(p=1,2, \cdots),
$$

with limit $f(z)$. Then if $f(z) \not \equiv S_{2 i}$ we have the following expansion:

$$
f(z)=\mathfrak{B}_{2 i}+z^{2 i}\left\{\frac{c_{1}^{(i)}}{z}-\frac{c_{2}^{(i)}}{z^{2}}-\frac{c_{3}^{(i)}}{z^{3}}-\cdots\right\},
$$

which is convergent for $|z|>R$, where $R$ is the distance from the origin to the most remote pole of $f(z)$, and is always finite. The numbers $c_{k}^{(i)}$ are given by (80), and thus, for every $k$,

$$
c_{2 k}^{(i)}-c_{2 k-1}^{(i)} z+\cdots-c_{1}^{(i)} z^{2 k-1}+c_{2 i} z^{2 k}-\cdots
$$

is an extension of $\mathfrak{P}^{2 i}(z)$ of order $k$.

9. An existence theorem for Case II. We shall apply the work of the preceding paragraph to show that, in Case II, for some $i$ the files $S_{-1}, S_{1}$, $S_{2}, \cdots, S_{2 i}$ may have a common limit while the succeeding odd files, $S_{2 i+1}$, $S_{2 i+3}, S_{2 i+5}, \cdots$, may either converge or diverge. To construct such examples, suppose $\mathfrak{B}(z)$ to be indeterminate, and let all the odd files of the associated Padé table be divergent. Let us now form the series (82) taking $k=i$. The series so formed will be determinate by Theorem 10, and in the corresponding Padé table, $S_{-1}, S_{0}, \cdots, S_{2 i}$ will be convergent, while the subsequent odd files must clearly diverge. If, on the other hand, the odd files in the table for $\mathfrak{B}(z)$ had been convergent, the same would have been true in the table for the constructed series. 
If, in Case II, $S_{2 i+2}$ is the first file different from $S_{2 i}(i \geqq 0)$, then $S_{2 i+2}$ is a meromorphic function of $1 / z$. The like is true of $S_{2 i+1}$ when the latter is convergent. It is readily shown that the common limit, $S_{0}$, of the preceding files is a meromorphic function of $1 / z$.

In fact, the series $\mathfrak{P}^{2 i+2}(z)$, being indeterminate, may be extended indefinitely. One such set of extensions is made up of the determinate series $\mathfrak{P}^{2 i-2 k}(z), k=0,1,2, \cdots, i$.

Now the function $m^{2 i+2}\left(z^{\prime}, t\right)$ connected with $\mathfrak{P}^{2 i+2}(z)$ may be written in the form

$$
\sum_{\nu=-\infty}^{+\infty} \frac{N_{\nu}(t)}{z^{\prime}+\lambda_{\nu}(t)}
$$

Let us take $t=c_{2 i+1}(\mathrm{cf} .(79))$, and consider the function

$$
\psi_{1}(u)=\int_{-\infty}^{u} \frac{d \psi^{2 i+2}\left(u, c_{2 i+1}\right)}{u^{2 i+2}} .
$$

Clearly the integrals

all exist and

$$
\int_{-\infty}^{+\infty} u^{i} d \psi_{1}(u)=c_{i}^{\prime} \quad(i=0,1,2, \cdots)
$$

$$
c_{i}^{\prime}=c_{i}, i=1,2, \cdots, c_{0}^{\prime}=c_{0}+\omega, \omega \geqq 0 .
$$

It follows that

$$
S_{0}=\omega+\frac{1}{z} \int_{-\infty}^{+\infty} \frac{d \psi_{1}(u)}{z^{\prime}+u}=\omega+\frac{1}{z} \sum_{v=-\infty}^{+\infty} \frac{N_{\nu}}{\left(\lambda_{v}\right)^{2 i+2}\left(z^{\prime}+\lambda_{v}\right)},
$$

where we have written $N_{\nu}, \lambda_{\nu}$ for $N_{\nu}\left(c_{2 i+1}\right), \lambda_{\nu}\left(c_{2 i+1}\right)$, respectively. Hence $S_{0}$ is a meromorphic function of $z^{\prime}=1 / z$.

NORTHWESTERN UNIVERSITY, Evanston, Ill. 